\title{
Downregulation of IncRNA CASC2 by microRNA-21 increases the proliferation and migration of renal cell carcinoma cells
}

\author{
YUNJIE CAO ${ }^{*}$, RENFANG XU* ${ }^{*}$ XIANLIN XU*, \\ YAOJUN ZHOU, LI CUI and XIAOZHOU HE
}

\begin{abstract}
Department of Urology, The Third Affiliated Hospital of Soochow University, Changzhou, Jiangsu 213003, P.R. China
\end{abstract}
Received June 21, 2015; Accepted April 21, 2016

DOI: $10.3892 / \mathrm{mmr} .2016 .5337$

\begin{abstract}
Several long non-coding RNAs (lncRNAs) have been identified that may have a crucial role in tumor progression and metastasis. The lncRNA cancer susceptibility candidate 2 (CASC2) has previously been reported to act as a tumor suppressor gene in glioma and colorectal cancer. However, the expression and function of CASC2 in renal cell carcinoma (RCC) remains to be elucidated. The present study confirmed that CASC2 was downregulated in human RCC tissues and human RCC cell lines (786-O and A498). Restoration of CASC2 expression via transfection with a pcDNA3.1(+)-CASC2 vector was able to inhibit cell proliferation and migration in 786-O and A498 cells, as compared with in the cells transfected with a pcDNA3.1(+) empty vector. MicroRNA-21 (miR-21) has been reported to be upregulated in human RCC tissues and cell lines, and is associated with the malignant progression of RCC. In the present study, bioinformatics analysis and dual-luciferase reporter assays confirmed that CASC2 was a direct target gene of miR-21. miR-21 was able to decrease the expression of CASC 2 in 786-O and A498 cells. Furthermore, overexpression of miR-21 partly abrogated CASC2-mediated inhibition of 786-O and A498 cell proliferation and migration. The present study provides evidence indicating that CASC2 targeted by miR-21 acts as a tumor suppressor in RCC. Therefore, CASC2 may be considered a novel target for the diagnosis and treatment of RCC.
\end{abstract}

\section{Introduction}

Renal cell carcinoma (RCC) is a type of highly metastasized tumor that accounts for $3 \%$ of all malignancies in adults. RCC

Correspondence to: Professor Xiaozhou He, Department of Urology, The Third Affiliated Hospital of Soochow University, 185 Juqian Street, Changzhou, Jiangsu 213003, P.R. China

E-mail: 13775188220@126.com

*Contributed equally

Key words: cancer susceptibility candidate 2, renal cell carcinoma, microRNA-21, suppressor has the highest rate of lethality out of all urological malignancies $(1,2)$. In the United States, there are $\sim 65,000$ new cases of RCC and $\sim 14,000$ cases of RCC-associated mortality annually (3). Despite the development of novel therapeutic drugs, it remains difficult to treat patients with metastatic RCC and prognostic improvements are rarely accomplished (4). Therefore, the development of a more effective therapy for $\mathrm{RCC}$ is required.

The long non-coding RNA (lncRNA) cancer susceptibility candidate 2 (CASC2), which is located at chromosome 10q26, was initially reported to be downregulated in endometrial cancer, where it acted as a tumor suppressor gene (5). Previous studies have demonstrated that exogenous expression of CASC2 significantly inhibits the growth of undifferentiated endometrial cancer cells and suppresses glioma cell metastasis $(6,7)$. However, little is currently known regarding the expression and function of CASC2 in RCC.

MicroRNAs (miRNAs) are non-coding RNA molecules (length, 22 nucleotides) which serve important regulatory roles in various biological processes, including proliferation, migration, invasion, apoptosis and cell cycle distribution (8). Emerging evidence suggests that miRNAs are aberrantly expressed in various types of human cancer, and are involved in cancer initiation, development and metastasis (9). miRNAs may suppress translation or induce mRNA cleavage by binding to the 3'-untranslated region (UTR) of target mRNA. As well as protein-coding genes, lncRNAs are novel targets of miRNAs (10).

The current study demonstrated that CASC2 is lowly expressed in RCC tissues and cell lines, suggesting that CASC2 acts as a tumor suppressor gene that may inhibit RCC cell proliferation and migration. Additionally, miRNA (miR)-21 decreased CASC 2 expression in a sequence-specific manner. Thus, the downregulation of CASC 2 by miR-21 may account for CASC2-mediated promotion of RCC cell proliferation and migration.

\section{Materials and methods}

Patient samples. A total of $32 \mathrm{RCC}$ tissues and paired normal tissues were collected from the Third Affiliated Hospital of Soochow University (Changzhou, China). Informed consent was obtained from all patients. The present study was approved by the Institutional Review Board of the Third Affiliated 
Hospital of Soochow University. All samples were frozen in liquid nitrogen immediately after surgery. The clinical information and pathological characteristics of the 32 patients with $\mathrm{RCC}$ are presented in Table I.

Cell culture. The 786-O and A498 human RCC cell lines, and human embryonic kidney (HEK) 293 cells were obtained from the American Type Culture Collection (Manassas, VA, USA). Cells were cultured in Dulbecco's modified Eagle's medium (Invitrogen; Thermo Fisher Scientific, Inc., Waltham, MA, USA) supplemented with $10 \%$ fetal bovine serum (Invitrogen; Thermo Fisher Scientific, Inc.), $1 \% 100 \mathrm{U} / \mathrm{ml}$ penicillin and $1 \% 100 \mathrm{mg} / \mathrm{ml}$ streptomycin sulfate (Sigma-Aldrich, St. Louis, MO, USA) at $37^{\circ} \mathrm{C}$ in a humidified incubator containing $5 \%$ $\mathrm{CO}_{2}$.

Cell transfection. Human CASC2 gene (NR_026939) was cloned into a pcDNA3.1(+) vector (Thermo Fisher Scientific, Inc.). CASC2 was amplified by polymerase chain reaction (PCR), then the PCR products were double-digested by restriction endonucleases of PmeI and NotI (Takara Bio, Inc., Otsu, Japan). Subsequently, the digested products were sub-cloned into the PmeI and NotI sites of the psiCHECK-2 luciferase vector. An empty vector served as a negative control (NC). The miR-21 mimics, 5'-UAGCUUAUCAGACUGAUG UUGA-3', and NC mimics, 5'-CAGUACUUUUGUGUAGUA CAA-3' were obtained from Shanghai GenePharma Co., Ltd. (Shanghai, China). pcDNA3.1(+)-CASC2 over-expression vector $(2 \mu \mathrm{g})$ or $2 \mu \mathrm{g}$ pcDNA3.1(+) empty vector was transfected into 786-O or A498 cells, and 200 pmol miR-21 mimics or NC mimics were transfected into 786-O or A498 cells. The 786-O and A498 cells were transfected using Opti-MEM and Lipofectamine 2000 reagents (Invitrogen; Thermo Fisher Scientific, Inc.) once the cells reached 50-70\% confluence, according to the manufacturer's protocols.

Reverse transcription-quantitative PCR (RT-qPCR). Total RNA of each sample (tissues and cells) was extracted with TRIzol Reagent (Invitrogen; Thermo Fisher Scientific, Inc.) according to the manufacturer's protocols. Synthesis of cDNA with reverse transcriptase was performed with the PrimeScript RT Enzyme Mix I kit (Takara Bio, Inc.). The reaction mixtures were incubated at $37^{\circ} \mathrm{C}$ for $60 \mathrm{~min}, 95^{\circ} \mathrm{C}$ for $5 \mathrm{~min}$ and then held at $4^{\circ} \mathrm{C}$. MiScript SYBR Green PCR kit (Qiagen, Inc., Valencia, CA, USA) was used to conduct a qPCR analysis. The sequences of the primers (Invitrogen; Thermo Fisher Scientific, Inc.) used were as follows: miR-21, forward 5'-TTGACTGTTGAATCTCATGGCAA-3', reverse primers were provided by the miScript SYBR Green PCR kit; CASC2, forward 5'-TACAGGACAGTCAGTGGT GGTA-3', reverse 5'-ACATCTAGCTTAGGAATGTGGC-3'; and human GAPDH (which served as an internal control), forward 5'-TCAAGAAGGTGGTGAAGCA-3' and reverse 5'-AGGTGGAGGAGTGGGTGT-3'. The qPCR reaction was set up in a total volume of $20 \mu \mathrm{l}$, consisting of $10 \mu \mathrm{l}$ MiScript SYBR Green PCR Mix, $2 \mu \mathrm{l}$ primers (forward and reverse), $1 \mu \mathrm{l}$ cDNA template and $7 \mu \mathrm{l}$ RNase-free water. The reactions were performed as follows: $95^{\circ} \mathrm{C}$ for $15 \mathrm{~min}$, followed by 40 cycles at $95^{\circ} \mathrm{C}$ for $10 \mathrm{sec}, 60^{\circ} \mathrm{C}$ for $30 \mathrm{sec}$ and $72^{\circ} \mathrm{C}$ for $20 \mathrm{sec}$, using an Applied Biosystems 7900 HT PRISM Real-time PCR
Table I. Clinical characteristics of 32 patients with renal cell carcinoma.

\begin{tabular}{lc}
\hline Characteristic & Number \\
\hline Age (years) & \\
$\geq 50$ & 18 \\
$<50$ & 14 \\
Gender & \\
Male & 23 \\
Female & 9 \\
Histological type & \\
Clear cell renal cell carcinoma & 25 \\
Papillary renal cell carcinoma & 7 \\
Stage & \\
T1a & 8 \\
T1b & 12 \\
T2 & 9 \\
T3 & 2 \\
T4 & 1 \\
Fuhrman grade & $3(\mathrm{~T} 1 \mathrm{a}), 3(\mathrm{~T} 1 \mathrm{~b}), 2(\mathrm{~T} 3), 1(\mathrm{~T} 4)$ \\
G1 & 0 \\
G2 & \\
G3 & $2(\mathrm{~T} 1 \mathrm{a}), 4(\mathrm{~T} 1 \mathrm{~b}), 5(\mathrm{~T} 1 \mathrm{~b})$, \\
G4 & $6(\mathrm{~T} 2)$ \\
\hline
\end{tabular}

system (Applied Biosystems; Thermo Fisher Scientific, Inc.). The PCR results were calculated using the $\Delta \Delta \mathrm{Cq}$ method (11).

In vitro cell proliferation assay. Proliferation of 786-O and A498 cells was measured using the 3-(4,5-dimethylthiazol-2-yl)-2,5-diphenyltetrazolium bromide (MTT) assay (Sigma-Aldrich). The cells $\left(\sim 2 \times 10^{4}\right)$ were seeded into a 96-well culture plate $24 \mathrm{~h}$ prior to transfection. After $0,24,48$ or $72 \mathrm{~h}$ of transfection, $20 \mu \mathrm{l}$ MTT ( $5 \mathrm{mg} / \mathrm{ml}$ ) was added to each well, and the plates were incubated for $3 \mathrm{~h}$ at $37^{\circ} \mathrm{C}$. Subsequently, the precipitate was solubilized with $150 \mu 1$ dimethyl sulfoxide (Sigma-Aldrich), and absorbance was measured at a wavelength of $450 \mathrm{~nm}$ using an enzyme-linked immunosorbent assay microplate reader (model 3550; Bio-Rad Laboratories, Inc., Hercules, CA, USA). Experiments were repeated at least three times.

In vitro cell migration scratch assay. $786-\mathrm{O}$ and $\mathrm{A} 498$ cells were transfected with pcDNA3.1(+)-CASC2 vector or pcDNA3.1(+) empty vector, and miR-21 mimics or NC mimics. A total of $6 \mathrm{~h}$ post-transfection, a cell-scratch spatula was used to make a scratch in the cell monolayer. The cells were then washed three times with phosphate-buffered saline and were incubated at $37^{\circ} \mathrm{C}$ for $24 \mathrm{~h}$. Images of the scratches were captured using a digital camera system (Leica DC 350FX; Leica Microsystems, Wetzlar, Germany) after the cells had been incubated for 0 and $24 \mathrm{~h}$. The software program MIAS-2000 (Leica Microsystems) was used to determine the 
A

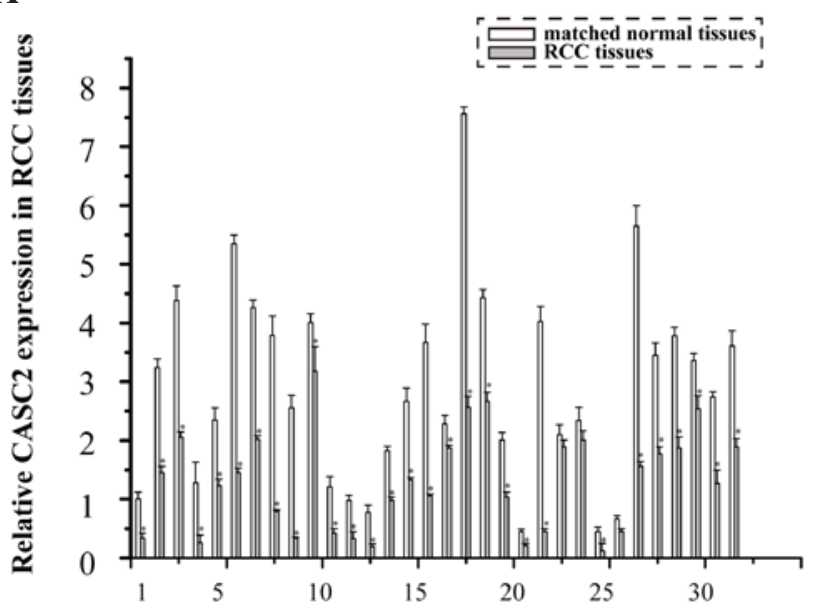

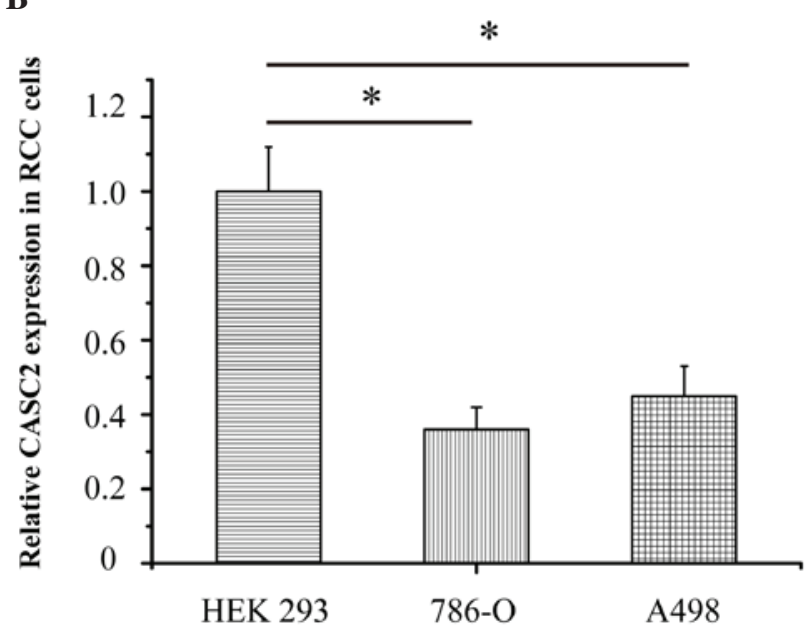

Figure 1. Cancer susceptibility candidate 2 (CASC2) was markedly downregulated in renal cell carcinoma (RCC) tissues and cell lines, as determined by quantitative polymerase chain reaction. (A) Expression levels of CASC2 were significantly downregulated in 32 RCC tissues compared with in matched normal tissues. (B) CASC2 expression was lower in 786-O and A498 cells compared with in human embryonic kidney HEK 293 cells. Glyceraldehyde 3-phosphate dehydrogenase was used as an internal reference. Data are presented as the mean \pm standard deviation. ${ }^{*} \mathrm{P}<0.05$.

distance of migration. The experiments were performed in triplicate, repeated at least three times, and were analyzed in a double-blind fashion by at least two observers.

Bioinformatics prediction. The potential miR-21 binding sites of CASC2 predicted by computer-aided algorithms were obtained from miRanda (http://www.microrna.org) and miRcode (http://www.mircode.org).

Dual-luciferase reporter assay. The RNA sequence of wild-type CASC2 (CASC2-WT) was amplified by PCR, and was inserted between the PmeI and NotI restriction sites in the 3'-UTR of the hRluc gene in the psiCHECK-2 luciferase vector (Promega Corporation, Madison, WI, USA). The primer sequences for CASC2 (Invitrogen; Thermo Fisher Scientific, Inc.) were as follows: Forward 5'-AGCGGGCTGCAGGGC TGCGGGCGCT-3' and reverse 5'-TTGATTTAAAGTAAT TAGCACATTC-3'. CASC2 RNA sequence with mutations in the putative binding site (CASC2-MUT) was also inserted into the hRluc gene in the psiCHECK-2 luciferase vector. CASC2-MUT was chemically synthesized by Shanghai GenePharma Co., Ltd. All recombinant plasmids were verified by DNA sequencing analysis.

For the dual-luciferase reporter assay, 786-O cells were seeded into 24 -well plates, and were co-transfected with $0.8 \mu \mathrm{g}$ CASC2-WT or $0.8 \mu \mathrm{g}$ CASC2-MUT plasmids and 80 pmol miR-21 mimics or 80 pmol NC mimics using Lipofectamine 2000. A total of $24 \mathrm{~h}$ post-transfection, firefly and Renilla luciferase activities were detected using the GloMax ${ }^{\circledR}$-Multi Jr Single Tube Multimode Reader (Promega Corporation), according to the Dual-Luciferase Reporter Assay system (Promega Corporation). All experiments were performed in triplicate and repeated at least three times.

Statistical analysis. Experimental data are presented as the mean \pm standard deviation, and were analyzed using SPSS 17.0 software (SPSS Inc., Chicago, IL, USA). Data were analyzed using Student's t-test or one-way analysis of variance and the
Student-Newman-Keuls method. $\mathrm{P}<0.05$ was considered to indicate a statistically significant difference.

\section{Results}

CASC2 is markedly downregulated in RCC tissues and cell lines, as determined by qPCR analysis. The expression levels of CASC2 were detected in 32 RCC specimens using qPCR. The expression levels of CASC2 were significantly downregulated in the $32 \mathrm{RCC}$ tissues compared with in the adjacent normal tissues (Fig. 1A; $\mathrm{P}<0.05$ ). The expression levels of CASC2 were also lower in the 786-O and A498 cell lines compared with in the human embryonic kidney HEK 293 cells (Fig. 1B; $\mathrm{P}=0.017$ and $\mathrm{P}=0.033$, respectively). These data suggest that CASC2 may function as a tumor suppressor gene in RCC development.

CASC2 inhibits 786-O and A498 cell proliferation and migration in vitro. To explore the function of CASC2 in RCC cell proliferation and migration, MTT and wound scratch assays were performed in 786-O and A498 cell lines. Post-transfection with the pcDNA3.1(+)-CASC2 overexpression vector, the optical density (OD) of 786-O cells revealed that relative cell proliferation was significantly decreased by $8.21 \%$ at $24 \mathrm{~h}, 14.22 \%$ at $48 \mathrm{~h}$ and $21.1 \%$ at $72 \mathrm{~h}(\mathrm{P}=0.001)$; the OD of A498 cells also demonstrated that proliferation was decreased by $6.36 \%$ at $24 \mathrm{~h}, 12.46 \%$ at $48 \mathrm{~h}$ and $16.32 \%$ at $72 \mathrm{~h}(\mathrm{P}=0.005$; Fig. 2A). Images of the wound scratches were captured at 0 and $24 \mathrm{~h}$ post-transfection; overexpression of CASC2 markedly inhibited the migration of 786-O and A498 cells. The distance of migration is presented in Fig. 2B. The relative rates of migration were $32 \%$ in $786-\mathrm{O}$ cells and $47 \%$ in A498 cells ( $\mathrm{P}=0.007$ and $\mathrm{P}=0.009$, respectively). These results indicate that $\mathrm{CASC} 2$ inhibits the proliferation and migration of RCC cells.

CASC2 is a direct target gene of miR-21 in RCC cells. Previous studies have reported that IncRNAs may act as competing 
A

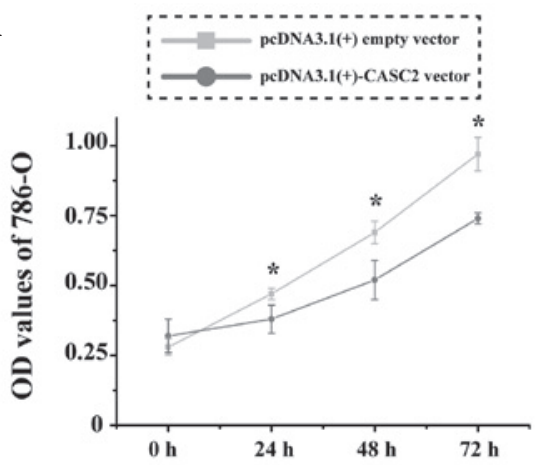

B
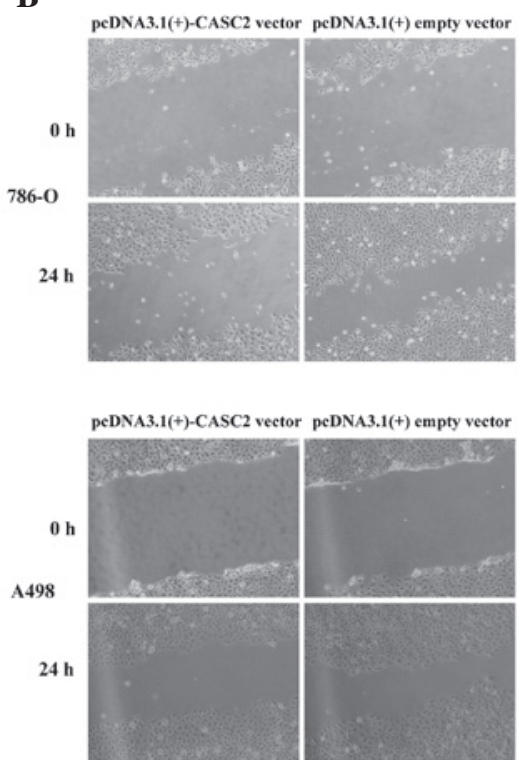
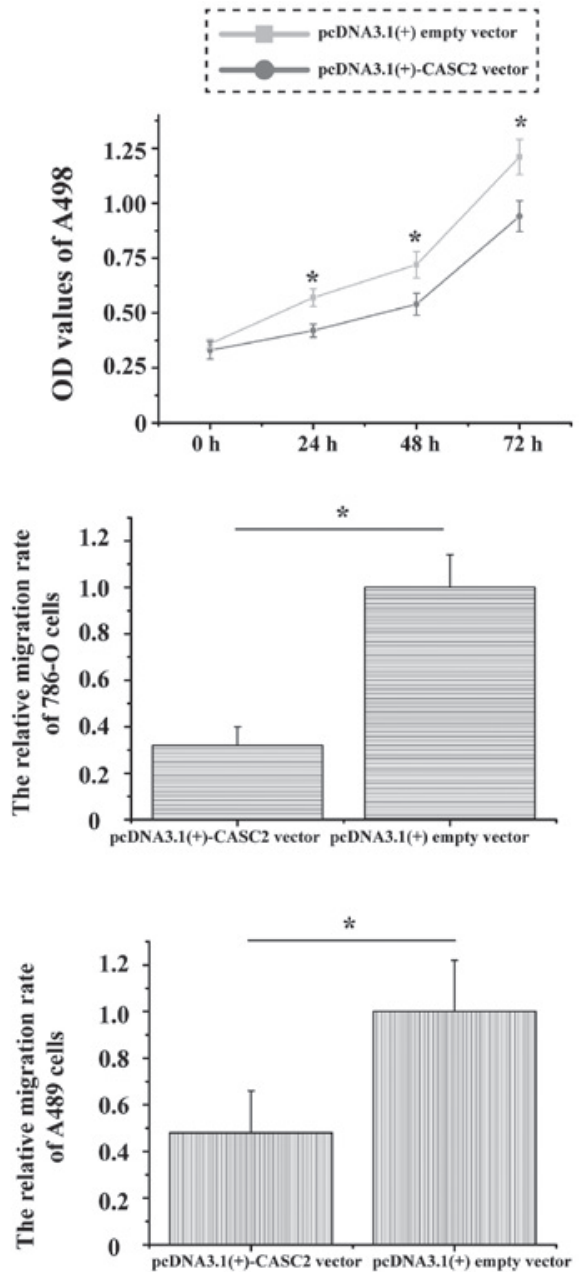

Figure 2. Cancer susceptibility candidate 2 (CASC2) inhibited 786-O and A498 cell proliferation and migration in vitro. (A) pcDNA3.1(+)-CASC2 vector or pcDNA3.1(+) empty vector-transfected 786-O and A498 cells were subjected to the 3-(4,5-dimethylthiazol-2-yl)-2,5-diphenyltetrazolium bromide assay. (B) A wound scratch assay was performed in 786-O and A498 cells post-transfection with pcDNA3.1(+)-CASC2 vector or pcDNA3.1(+) empty vector. Data are presented as the mean \pm standard deviation. ${ }^{*} \mathrm{P}<0.01$.

\section{A}

CASC2-WT 5'-AAUUGACAUCUAAACAUAAGCUGAGAGG -3,
miR-21 5'-AGUUGUAGUCAGACUAUUCGAU -3,
CASC2-MUT 5'- AAUUGACAUCUAAACCGUUACGAGAGG -3,
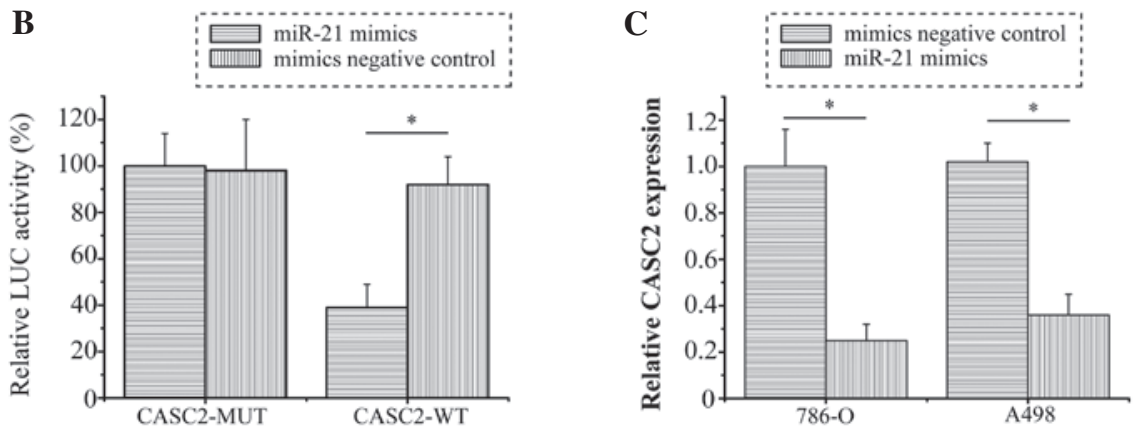

Figure 3. Cancer susceptibility candidate 2 (CASC2) was a direct target gene of microRNA (miR)-21 in renal cell carcinoma cells. (A) Predicted binding site between miR-21 and CASC2 is shown. (B) Luciferase (LUC) activity assays were conducted in 786-O cells, following co-transfection with CASC2-wild-type (WT) or CASC2-mutant (MUT) recombinant vectors and miR-21 mimics or negative control mimics. (C) Quantitative polymerase chain reaction analysis was used to measure the expression levels of CASC2 post-transfection with miR-21 mimics or negative control mimics in 786-O and A498 cells. Data are presented as the mean \pm standard deviation. ${ }^{*} \mathrm{P}<0.01$. 
A

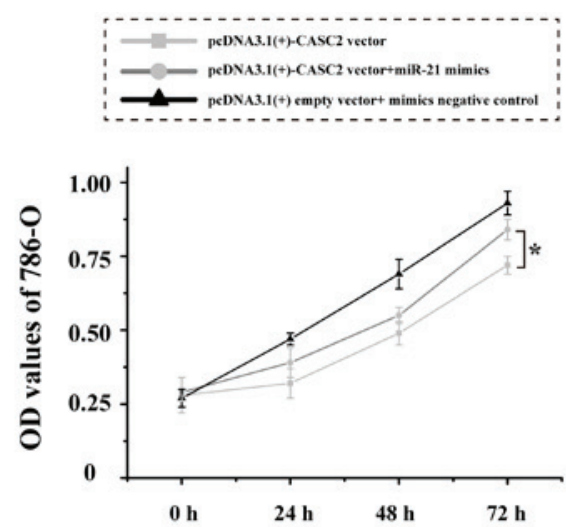

B
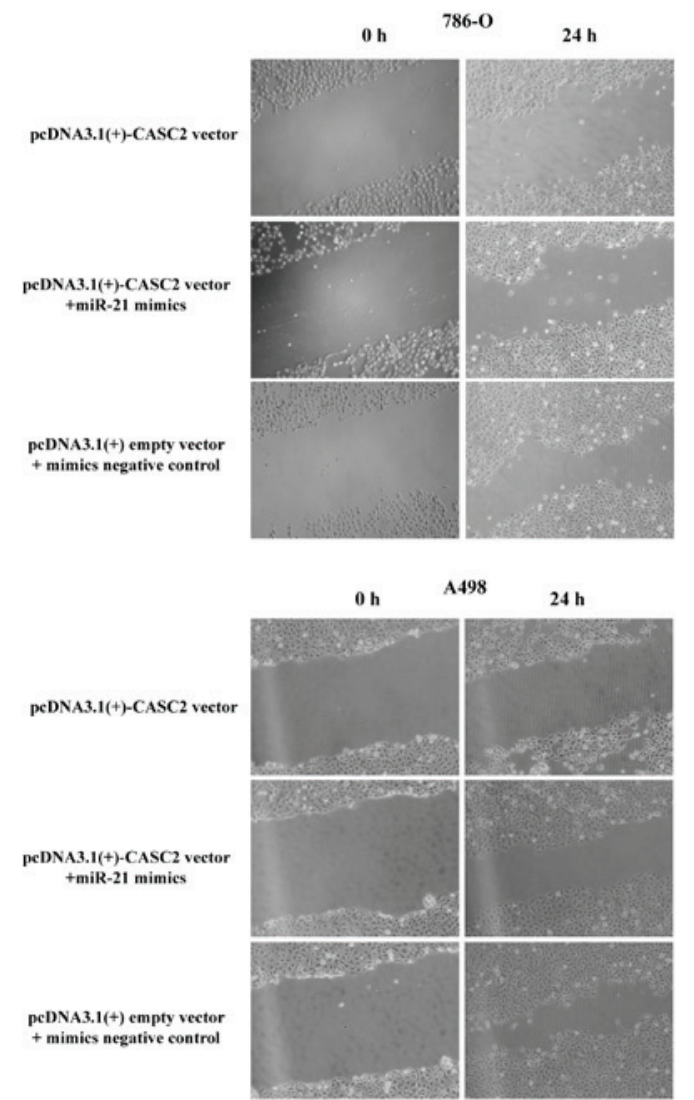
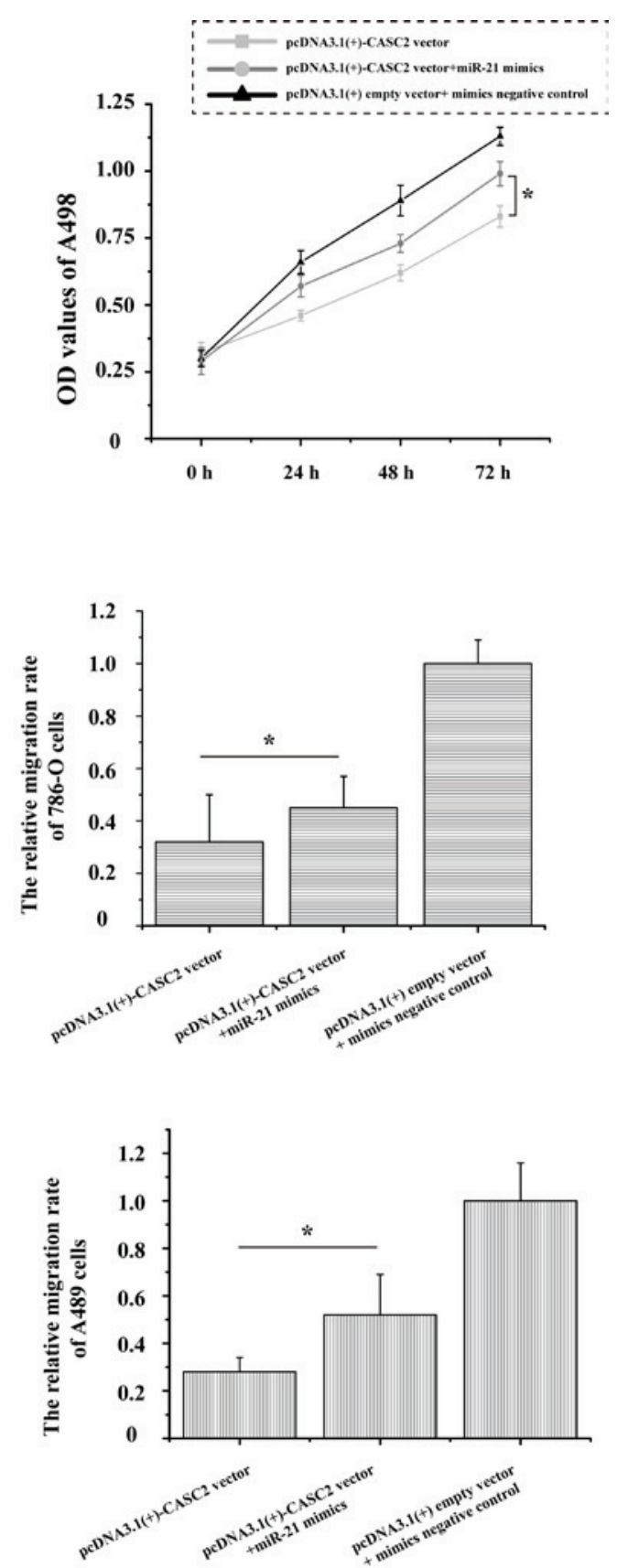

Figure 4. Restoration of microRNA (miR)-21 expression partly abrogated cancer susceptibility candidate 2 (CASC2)-induced inhibitory effects on renal cell carcinoma cells. (A) Cell proliferation was measured by 3-(4,5-dimethylthiazol-2-yl)-2,5-diphenyltetrazolium bromide assay in 786-O and A498 cells transfected with pcDNA3.1(+)-CASC2 vector or miR-21 mimics + pcDNA3.1(+)-CASC2 vector or pcDNA3.1(+) empty vector. (B) Wound scratch assay was conducted in 786-O and A498 cells transfected with pcDNA3.1(+)-CASC2 vector or miR-21 mimics + pcDNA3.1(+)-CASC2 vector or pcDNA3.1(+) empty vector. Data are presented as the mean \pm standard deviation. ${ }^{*} \mathrm{P}<0.01$.

endogenous RNAs or as molecular sponges that modulate the concentration and biological function of miRNAs $(12,13)$. Bioinformatics analysis was performed in order to identify the potential targeted miRNA of CASC2. The results revealed that miR-21 binding sites were present in CASC2 (Fig. 3A). To further confirm whether CASC2 was a direct target of miR-21, a dual-luciferase reporter assay was conducted in 786-O cells. Consistent with expectations, the relative luciferase activity was significantly inhibited by miR-21 in the CASC2-WT vector-transfected cells $(\mathrm{P}=0.008)$, whereas miR-21 exhibited no inhibitory effect in cells transfected with the CASC2-MUT vector (Fig. 3B). Furthermore, as shown in Fig. 3C, the RNA expression levels of CASC2 were significantly downregulated in the cells transfected with miR-21 mimics compared with in the $\mathrm{NC}$ mimics group $(\mathrm{P}<0.001$ and $\mathrm{P}=0.005$ in $786-\mathrm{O}$ and A498 cells, respectively). These results suggest that CASC2 may be directly targeted by miR-21 in RCC.

Over-expression of miR-21 partly abrogates CASC2-induced inhibitory effects on RCC cells. CASC2 was identified as a direct target of miR-21 in RCC, as demonstrated by dual-luciferase reporter assays. The role of miR-21 in CASC2-induced inhibition on RCC cells remains unknown. As presented in Fig. 4A, the growth of 786-O and A498 cells was significantly 
increased $(\mathrm{P}=0.017$ and $\mathrm{P}=0.011$, respectively) following transfection with the pcDNA3.1(+)-CASC2 vector + miR-21 mimics as compared with the pcDNA3.1(+)-CASC2 vector. Additionally, over-expression of miR-21 and CASC2 could promote 786-O $(\mathrm{P}=0.042)$ and $\mathrm{A} 498(\mathrm{P}=0.025)$ cell migration when compared with over-expression of CASC2 (Fig. 4B). These results strongly indicate that over-expression of miR-21 in RCC cells partially reverses the inhibitory effects of CASC2 on cell proliferation and migration.

\section{Discussion}

Previous studies have demonstrated that the molecular mechanisms underlying carcinogenesis are not only associated with protein-coding genes but also with non-coding regulatory RNAs (14-16). Various IncRNAs have been identified to have a crucial role in regulating the development of cancer (17-19). Previous studies have revealed that several $\operatorname{lncRNAs}$ are aberrantly expressed in RCC $(20,21)$. Furthermore, several lncRNAs have been reported to be involved in regulating RCC metastasis, including proliferation, migration, invasion and apoptosis $(20,22)$.

Previous studies have reported that the downregulation of the IncRNA, CASC2 is associated with numerous types of carcinoma, including human endometrial cancer and gliomas (5-7). As a common type of urological cancer, the expression and function of CASC2 in RCC has not yet, to the best of our knowledge, been reported. The present study illustrated the expression and role of CASC2 in RCC development. Consistent with our hypotheses, the results indicated that the expression levels of CASC2 were significantly lower in RCC specimens and cell lines, compared with that in adjacent normal tissue samples and human HEK 293 cells. Furthermore, restoration of CASC2 expression in 786-O and A498 cells inhibited cell proliferation and migration. These results suggested that dysregulation of CASC2 exerts a suppressive role in RCC development.

In a previous study, miR-21 was demonstrated to be upregulated in RCC tissues and cell lines (23). miR-21 has been shown to promote cell transformation, proliferation and metastasis in RCC by targeting the tumor suppressor programmed cell death protein 4 (24). In addition, miR-21 may influence RCC cell proliferation by regulating nuclear factor- $\mathrm{\kappa B}$-mediated cyclin D1 expression (25). In the present study, the results of the bioinformatics analysis and dual-luciferase reporter assay confirmed that CASC2 was a direct target gene of miR-21. Furthermore, upregulated miR-21 expression was able to suppress CASC2 expression in 786-O and A498 cells, thus suggesting that CASC2 is a direct target gene of miR-21 in RCC.

To further verify the speculation that miR-21 functions by targeting CASC2 in RCC, synthetic miR-21 mimics were used to overexpress miR-21 in 786-O and A498 cells. miR-21 was able to partially reverse pcDNA3.1(+)-CASC2 vector-induced cell proliferation and migration inhibition. These results suggested that miR-21 has a key role inhibiting CASC2.

In conclusion, CASC2 acts as a tumor suppressor gene in RCC. CASC2 is directly targeted by miR-21, and ectopic $\mathrm{CASC} 2$ expression inhibits proliferation and migration of RCC cells. The present study may provide an improved understanding regarding the role of CASC2 in RCC development, which may be used to develop IncRNA-directed diagnostics and therapeutics against RCC.

\section{Acknowledgements}

The present study was supported by the Changzhou Health Research Program (grant no. CE20125025).

\section{References}

1. Rini BI, Campbell SC and Escudier B: Renal cell carcinoma. Lancet 373: 1119-1132, 2009.

2. Zhang WB, Pan ZQ, Yang QS and Zheng XM: Tumor suppressive miR-509-5p contributes to cell migration, proliferation and antiapoptosis in renal cell carcinoma. Ir J Med Sci 182: 621-627, 2013.

3. Siegel R, Naishadham D and Jemal A: Cancer statistics, 2013. CA Cancer J Clin 63: 11-30, 2013.

4. Redova M, Poprach A, Besse A, Iliev R, Nekvindova J, Lakomy R, Radova L, Svoboda M, Dolezel J, Vyzula R and Slaby O: MiR-210 expression in tumor tissue and in vitro effects of its silencing in renal cell carcinoma. Tumour Biol 34: 481-491, 2013.

5. Baldinu P, Cossu A, Manca A, Satta MP, Sini MC, Rozzo C, Dessole S, Cherchi P, Gianfrancesco F, Pintus A, et al: Identification of a novel candidate gene, CASC2, in a region of common allelic loss at chromosome 10q26 in human endometrial cancer. Hum Mutat 23: 318-326, 2004.

6. Baldinu P, Cossu A, Manca A, Satta MP, Sini MC, Palomba G, Dessole S, Cherchi P, Mara L, Tanda F and Palmieri G: CASC2a gene is down-regulated in endometrial cancer. Anticancer Res 27: 235-243, 2007.

7. Wang P, Liu YH, Yao YL, Li Z, Li ZQ, Ma J and Xue YX: Long non-coding RNA CASC2 suppresses malignancy in human gliomas by miR-21. Cell Signal 27: 275-282, 2015.

8. Yu Z, Chen D, Su Z, Li Y, Yu W, Zhang Q, Yang L, Li C, Yang S, $\mathrm{Ni}$ L, et al: MiR-886-3p upregulation in clear cell renal cell carcinoma regulates cell migration, proliferation and apoptosis by targeting PITX1. Int J Mol Med 34: 1409-1416, 2014.

9. Ha TY: MicroRNAs in human diseases: From cancer to cardiovascular disease. Immune Netw 11: 135-154, 2011.

10. Paraskevopoulou MD, Georgakilas G, Kostoulas N, Reczko M, Maragkakis M, Dalamagas TM and Hatzigeorgiou AG: DIANA-LncBase: Experimentally verified and computationally predicted microRNA targets on long non-coding RNAs. Nucleic Acids Res 41 (Database Issue): D239-D245, 2013.

11. Chen C, Ridzon DA, Broomer AJ, Zhou Z, Lee DH, Nguyen JT, Barbisin M, Xu NL, Mahuvakar VR, Andersen MR, et al: Real-time quantification of microRNAs by stem-loop RT-PCR. Nucleic Acids Res 33: e179, 2005.

12. Cesana M, Cacchiarelli D, Legnini I, Santini T, Sthandier O, Chinappi M, Tramontano A and Bozzoni I: A long noncoding RNA controls muscle differentiation by functioning as a competing endogenous RNA. Cell 147: 358-369, 2011.

13. Liu XH, Sun M, Nie FQ, Ge YB, Zhang EB, Yin DD, Kong R, Xia R, Lu KH, Li JH, et al: Lnc RNA HOTAIR functions as a competing endogenous RNA to regulate HER 2 expression by sponging miR-331-3p in gastric cancer. Mol Cancer 13: 92, 2014.

14. Qiao HP, Gao WS, Huo JX and Yang ZS: Long non-coding RNA GAS5 functions as a tumor suppressor in renal cell carcinoma. Asian Pac J Cancer Prev 14: 1077-1082, 2013.

15. Prensner JR and Chinnaiyan AM: The emergence of lncRNAs in cancer biology. Cancer Discov 1: 391-407, 2011.

16. Li Y and Wang X: Role of long noncoding RNAs in malignant disease (Review). Mol Med Rep 13: 1463-1469, 2016.

17. Salameh A, Lee AK, Cardó-Vila M, Nunes DN, Efstathiou E, Staquicini FI, Dobroff AS, Marchiò S, Navone NM, Hosoya $\mathrm{H}$, et al: PRUNE2 is a human prostate cancer suppressor regulated by the intronic long noncoding RNA PCA3. Proc Natl Acad Sci USA 112: 8403-8408, 2015. 
18. Li T, Xie J, Shen C, Cheng D, Shi Y, Wu Z, Deng X, Chen H, Shen B, Peng C, et al: Upregulation of long noncoding RNA ZEB1-AS1 promotes tumor metastasis and predicts poor prognosis in hepatocellular carcinoma. Oncogene 35: 1575-1584, 2016.

19. Liang WC, Fu WM, Wong CW, Wang Y, Wang WM, Hu GX, Zhang L, Xiao LJ, Wan DC, Zhang JF and Waye MM: The IncRNA H19 promotes epithelial to mesenchymal transition by functioning as miRNA sponges in colorectal cancer. Oncotarget 6: 22513-22525, 2015.

20. Hirata H,Hinoda Y,Shahryari V, Deng G,Nakajima K, TabatabaiZL, Ishii $\mathrm{N}$ and Dahiya R: Long noncoding RNA MALAT1 promotes aggressive renal cell carcinoma through Ezh2 and interacts with miR-205. Cancer Res 75: 1322-1331, 2015.

21. Song S, Wu Z, Wang C, Liu B, Ye X, Chen J, Yang Q, Ye H, Xu B and Wang L: RCCRT1 is correlated with prognosis and promotes cell migration and invasion in renal cell carcinoma. Urology 84: 730. e1-e7, 2014.
22. Wang L, Cai Y, Zhao X, Jia X, Zhang J, Liu J, Zhen H, Wang T, Tang X, Liu Y and Wang J: Down-regulated long non-coding RNA H19 inhibits carcinogenesis of renal cell carcinoma. Neoplasma 62: 412-418, 2015.

23. Lv L, Huang F, Mao H, Li M, Li X, Yang M and Yu X: MicroRNA-21 is overexpressed in renal cell carcinoma. Int J Biol Markers 28: 201-207, 2013.

24. Li X, Xin S, He Z, Che X, Wang J, Xiao X, Chen J and Song X: MicroRNA-21 (miR-21) post-transcriptionally downregulates tumor suppressor PDCD4 and promotes cell transformation, proliferation and metastasis in renal cell carcinoma. Cell Physiol Biochem 33: 1631-1642, 2014.

25. Bera A, Ghosh-Choudhury N, Dey N, Das F, Kasinath BS Abboud HE and Choudhury GG: NFkB-mediated cyclin D1 expression by microRNA-21 influences renal cancer cell proliferation. Cell Signal 25: 2575-2586, 2013. 